\title{
Depth resolution of piezoresponse force microscopy
}

\author{
Florian Johann, ${ }^{1, a)}$ Yongjun J. Ying, ${ }^{2}$ Tobias Jungk, ${ }^{1}$ Ákos Hoffmann, ${ }^{1}$ Collin L. Sones, ${ }^{2}$ \\ Robert W. Eason, ${ }^{2}$ Sakellaris Mailis, ${ }^{2}$ and Elisabeth Soergel ${ }^{1}$ \\ ${ }_{1}^{1}$ Institute of Physics, University of Bonn, Wegelerstraße 8, 53115 Bonn, Germany \\ ${ }^{2}$ Optoelectronics Research Centre, University of Southampton, Highfield, Southampton, \\ SO17 1BJ, United Kingdom
}

(Received 11 March 2009; accepted 8 April 2009; published online 28 April 2009)

Given that a ferroelectric domain is generally a three dimensional entity, the determination of its area as well as its depth is mandatory for full characterization. Piezoresponse force microscopy (PFM) is known for its ability to map the lateral dimensions of ferroelectric domains with high accuracy. However, no depth profile information has been readily available so far. Here, we have used ferroelectric domains of known depth profile to determine the dependence of the PFM response on the depth of the domain, and thus effectively the depth resolution of PFM detection. (C) 2009 American Institute of Physics. [DOI: 10.1063/1.3126490]

During the past decade piezoresponse force microscopy (PFM) has become a standard tool for the investigation of ferroelectric domains. ${ }^{1,2}$ This is mainly because of its ease of use (no specific sample preparation) combined with its capability for imaging ferroelectric domains with high lateral resolution of $<20 \mathrm{~nm} .{ }^{3}$ Furthermore, PFM is not limited to specific crystallographic orientations of the sample, and hence ferroelectric domains can be visualized with PFM on all faces of the crystal. ${ }^{4}$ Being an all-purpose analytical tool, and therefore advantageous with respect to many other relevant techniques used for the investigation of ferroelectric domains, ${ }^{5}$ it is often ignored that PFM produces twodimensional maps only of the domain patterns. The question that arises is: up to what depth below the surface is PFM sensitive? While some earlier attempts at addressing this problem were performed using thin films, ${ }^{6,7}$ to date, however, there are no reports on measurements using single crystals. Such samples are needed therefore as they uniquely allow for a defined domain configuration, and thus to quantitatively determine the depth resolution of PFM.

The goal of the investigations which are presented in this paper was to obtain a direct correlation between the depth of a surface domain ${ }^{8}$ and the corresponding contrast obtained in PFM measurements. The first challenge was thus to fabricate a sample with ferroelectric surface domains of known depth. A method that can produce such domains in lithium niobate is UV laser-induced inhibition of poling, ${ }^{9}$ a brief description of which is given here for clarity. It was found that irradiation of the $+z$ polar surface of lithium niobate crystals with UV laser radiation locally increases the coercive field. Hence, a preirradiated area of the crystal surface will maintain its original polarity after a subsequent uniform electric field poling step. The depth $d_{0}$ of those poling inhibited domains is of the order of a few microns, depending on the specific UV-writing conditions, such as the illuminating laser light (wavelength and intensity) and scan speed used. ${ }^{10}$ Linear ferroelectric domain tracks several millimeters long were produced by scanning the crystal sample in front of the focused laser beam.

${ }^{\text {a)} E l e c t r o n i c ~ m a i l: ~ j o h a n n @ p h y s i k . u n i-b o n n . d e . ~}$
In order to obtain surface domains of different depth $d_{0}$ the sample was wedge polished at a shallow angle $\left(\alpha=5^{\circ}\right)$. For a domain of $d_{0}=3 \mu \mathrm{m}$ depth we thereby obtained a smooth transition from domain depths of $0-3 \mu \mathrm{m}$ over a distance of $l=d_{0} / \sin \alpha \approx 35 \mu \mathrm{m}$. We then briefly etched the sample in hydrofluoric acid (HF) to enable subsequent scanning electron microscopy (SEM) imaging. Figure 1 shows a schematic of the cross sections of the wedge polished sample. In some cases a damaged region is observed in the center of the poling inhibited stripe. This is a consequence of the Gaussian profile of the irradiating UV laser beam where the high intensity portion of the laser beam can lead to localized melting of the surface. The melted region is then rapidly quenched producing a polycrystalline or amorphous layer with no net piezoelectric response.

PFM utilizes a scanning force microscope operated in contact mode with an additional voltage applied to the tip. The imaging of ferroelectric domains with PFM is based on the fact that ferroelectricity implies piezoelectricity, hence mapping the piezoelectric response of the crystal directly reflects its domain structure. To allow sensitive readout of the piezomechanical deformation of the material, an alternating voltage $U$ sin $\omega t$ is applied to the tip and lock-in detection is used for the measurements. A more detailed description of PFM can be found in Refs. 1, 4, 11, and 12 .

For the experiments we used a stand-alone scanning force microscope (SMENA, NT-MDT, Russia). Diamondcoated tips with a nominal radius of 50-70 nm (DCP11, NTMDT) were utilized. The voltage applied to the tip ( $\left.5 \mathrm{~V}_{\text {rms }}\right)$

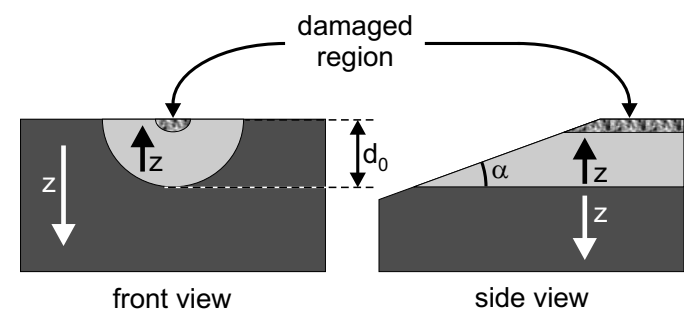

FIG. 1. Illustration of the cross sections of the sample used in the experiments. A lithium niobate crystal with a stripe surface domain (depth $d_{0}$ ) is wedge polished at an angle $\alpha$. At the center of the domain the crystal is damaged due to high laser irradiation during the fabrication process. 


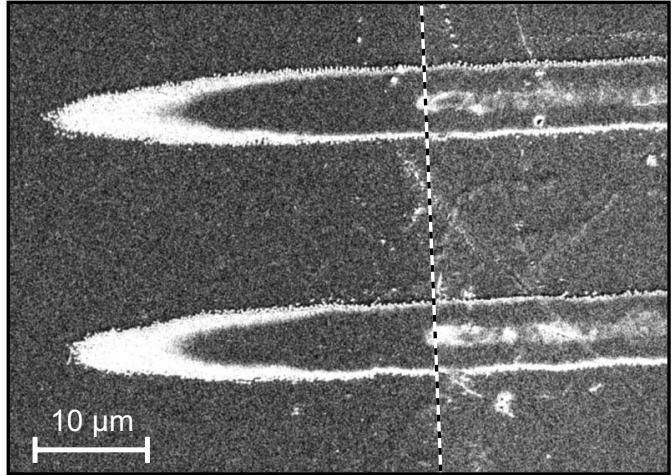

FIG. 2. HF-etched wedge-polished sample imaged with scanning electron microscopy. The dashed line indicates the position of the edge caused by the wedge polishing.

was directly provided by the lock-in amplifier (SRS 830, Stanford Research Systems).

Figure 2 shows an SEM image of a wedge-polished sample with two surface domains. In the right part of the image, the damaged region in the center of the domains is clearly visible. Furthermore, a bright halo around the domains can be observed. This feature is attributed to the imperfect boundary between the pole-inhibited domain and the surrounding bulk domain. Because the sample has been wedge-polished, thus gradually thinning the surface domain, the latter appears as a composite of nanodomains at its thinnest region, as shown in the schematic in Fig. 3(a). To verify this argument we recorded high-resolution PFM images at the tail end of a wedge-polished pole-inhibited domain [Fig. 3(b)]. Obviously the ferroelectric surface domain in this portion is no longer solid but a composite of many nanodomains.

Figure 4 shows the results from the scanning probe microscopy measurements of the whole wedge polished area, where topography (a) and piezoresponse (b) of the sample were recorded simultaneously. To reveal the topography of the HF-etched sample, the slope of the wedge has been subtracted from the image by data processing. The shape of the ferroelectric domain is the same as in Fig. 2. Its maximum depth was determined to be $d_{0}=l \sin \alpha=35.6 \mu \mathrm{m} \times \sin 5^{\circ}=3.1 \mu \mathrm{m}$. Compared with the topography (a) the PFM image in (b) shows some distinct features of the surface domain. For clarity a schematic of the PFM image is depicted in Fig. 4(c). Four areas showing different amplitudes in the PFM image are identified as follows: (A) the stripe associated with the central damaged region, (B) the area with a solid surface domain, corresponding to a $+z$-face, (C) the part where isolated nanodomains prevail, and (D) the surrounding area where the full PFM signal for

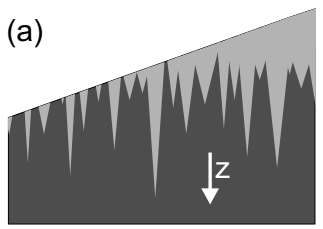

side view

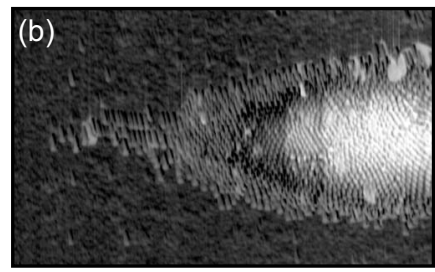

FIG. 3. Schematic (a) of the domain configuration at the limits of the poleinhibited surface domain. The termination of the domain is not sharp resulting in a grainy domain structure as it can be seen in the PFM image (b). Image size is $15 \times 9 \mu \mathrm{m}^{2}$.
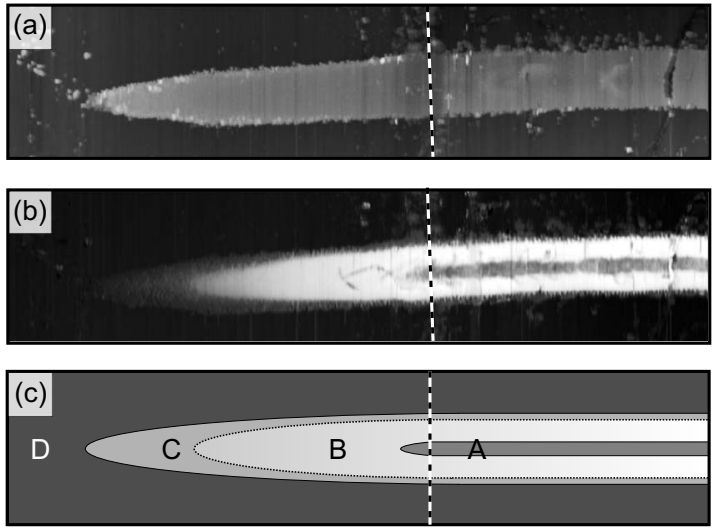

FIG. 4. Topography (a) and simultaneously recorded piezoresponse (b) of the wedge-polished sample shown in Fig. 2. Section (c) shows a schematic of the PFM image with four distinct areas marked. A: damaged region, B: full contrast PFM response corresponding to a $+z$-face, C: reduced PFM response, and D: surrounding uniform domain $(-z$-face $)$ area. The dashed lines indicate the position of the edge owing to wedge polishing. For representation purposes, the wedge has been subtracted from the topography image. Image size is $73 \times 16 \mu \mathrm{m}^{2}$.

the opposite orientation ( $-z$-face) is detected.

In order to determine the depth resolution of PFM measurements the dependence of the PFM contrast on the depth $d$ of the surface domain must be investigated. We therefore took scan-lines along the ferroelectric domain imaged in Fig. 4(b). Figure 5 shows two scan lines where one passes through the damaged area (black $\bullet$ ), while the other does not (gray $\times)$. The letters $(\mathrm{A}, \mathrm{B}, \mathrm{C}$, and $\mathrm{D})$ correspond to the regions identified in Fig. 4(c). Note that the presumably sharp change in the contrast between area $\mathrm{B}$ and $\mathrm{C}$ in Fig. 4(b) cannot be observed in the slope of the graphs in Fig. 5. However, these two regions can be distinguished when comparing the noise: in region $\mathrm{C}$ where we observed the nanodomains (Fig. 3) the data points fluctuate much more. In addition, at the intersection between $\mathrm{B}$ and $\mathrm{C}$ the curvature of the graph changes its sign. This again is consistent with our proposition of an uneven transition between the surface domain and the bulk domain, leading to nanodomain regions following sample wedge polishing. A theoretical model should therefore only reflect part B of the measured scan line.

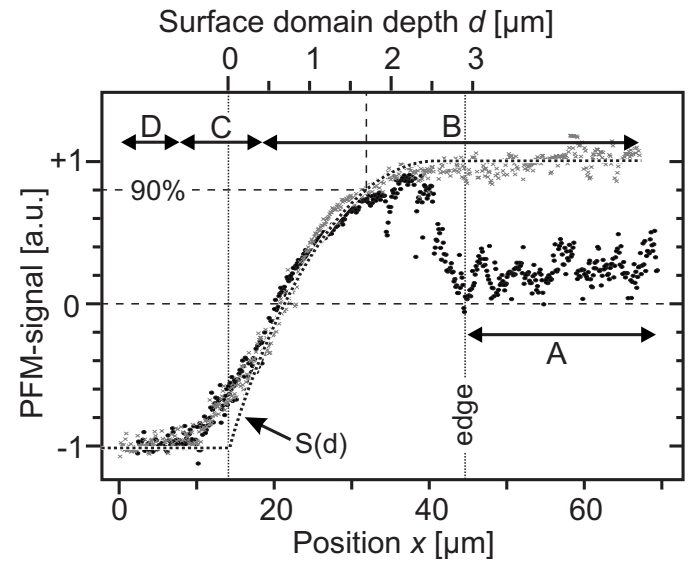

FIG. 5. Scan-lines across the PFM image of Fig. 4(b), one line passes through the damaged area (black $\bullet$ ) while the other line does not (gray $\times$ ). The letters indicate the areas shown in Fig. 4(c). The curve $S(d)$ is the result of the simulation. 
To obtain a reliable value for the depth resolution in PFM we calculated the expected depth dependence of PFM by means of a simplified model. We therefore approximated the spherical apex of the tip (radius $r$ ) by a point charge at the distance $r$ from the sample surface. The resulting piezomechanical deformation was then obtained by integrating all contributions of the sample within the volume of the crystal experiencing the electric field from the point charge. ${ }^{3}$ The result of our calculation can be seen in Fig. 5 where the curve $S(d)$ reflects favorably the measured slope within part $\mathrm{B}$, as it is expected from the considerations described above. The visible depth $d_{\mathrm{vis}}$ of PFM, i.e., the depth below the crystals surface where the contribution to the PFM signal has increased to $90 \%$ of that observed with bulk domains in a thick crystal, can be estimated to be $d_{\text {vis }} \approx 1.7 \mu \mathrm{m}$. Obviously PFM cannot provide any information about ferroelectric domains at depths $d>1.7 \mu \mathrm{m}$. In other words, surface domains with $d>d_{\text {vis }}$ cannot be distinguished from bulk domains by PFM.

It would, however, be useful to establish whether this measurement has a global rather than a material specific value. In order to investigate to what extent this result can be generalized to other ferroelectric materials ${ }^{13,14}$ apart from $\mathrm{LiNbO}_{3}$ we have considered two extreme cases of electrostatic interaction to simulate the interaction between the PFM tip and the surface: (a) parallel-plate capacitor configuration and (b) the point charge model. For case (a), the electric field inside the sample is homogeneous $\left(E_{z}=\rho / \varepsilon, \rho\right.$ being the surface charge density), thus while the field distribution does not depend on the material parameters, the strength of the field, however, is a function of the material. For the second case (b), where a point charge $q$ is located at a distance $r$ from the sample surface, the electric field $E_{z}$ inside the crystal, normal to the sample surface, can be written as follows: ${ }^{3}$

$$
E_{z}(x, y, z)=\frac{2 q \gamma}{1+\varepsilon_{\mathrm{eff}}} \frac{z+r}{\left[x^{2}+y^{2}+(z+r)^{2}\right]^{3 / 2}},
$$

whereby $\gamma=\sqrt{\varepsilon_{z} / \varepsilon_{r}}$ and $\varepsilon_{\text {eff }}=\sqrt{\varepsilon_{z} \varepsilon_{r}}$ and $\varepsilon_{z}$ and $\varepsilon_{r}$ are the dielectric constants of the material $\left(\varepsilon_{z}\right.$ in $z$-direction and $\varepsilon_{r}$ perpendicular to z). As can be seen from Eq. (1), again only the amplitude of the electric field depends on the material properties but not its spatial distribution. Although the actual situation of the PFM tip in contact with the surface cannot be accurately described by either case [(a) or (b)] it is expected that, by common sense interpolation of the two extreme cases calculated above, the actual electric field inside the sample is also independent on the material parameters. Con- sequently, the visible depth for ferroelectric domains in bulk crystals is $d_{\mathrm{vis}} \approx 1.7 \mu \mathrm{m}$, irrespective of the material. Although this depth can be considered to be very small in terms of bulk crystals, this value becomes important when ferroelectric domain patterns in thin films are investigated. Not only is the thickness of the film smaller (typically several $100 \mathrm{~nm}$ ) than $d_{\text {vis }}$ but also the single crystalline grains are of the order of $<100 \mathrm{~nm}$. PFM images show therefore averages of several grains lying one above the other, which is why any quantitative conclusions from PFM measurement on such films is challenging. Note that if a lower lateral resolution can be tolerated, the visible depth $d_{\text {vis }}$ can be increased by using a tip with larger radius $r$. Unfortunately the visible depth cannot be increased by applying a higher voltage $U \sin \omega t$ to the tip as a change in the voltage only changes the amplitude of the signal but not the shape of the curve.

In conclusion, we have accomplished a detailed analysis of the depth resolution of PFM. For the case of lithium niobate we have determined the visible depth to be $\approx 1.7 \mu \mathrm{m}$. From basic considerations we concluded that this depth is universal for all bulk crystals, irrespective of the material.

Financial support from the Deutsche Telekom AG, and the European Union, under the STREP 3D-DEMO is gratefully acknowledged.

\footnotetext{
${ }^{1}$ Nanoscale Characterisation of Ferroelectric Materials, 1st ed., edited by M. Alexe and A. Gruverman (Springer, New York, 2004).

${ }^{2}$ T. Jungk, A. Hoffmann, and E. Soergel, Appl. Phys. Lett. 89, 163507 (2006).

${ }^{3}$ T. Jungk, A. Hoffmann, and E. Soergel, New J. Phys. 10, 013019 (2008).

${ }^{4}$ T. Jungk, A. Hoffmann, and E. Soergel, New J. Phys. 11, 033029 (2009).

${ }^{5}$ E. Soergel, Appl. Phys. B: Lasers Opt. 81, 729 (2005).

${ }^{6}$ X. M. Lu, F. Schlaphof, S. Grafström, C. Loppacher, and L. M. Eng, Appl. Phys. Lett. 81, 3215 (2002).

${ }^{7}$ L. M. Eng, S. Grafström, C. Loppacher, X. M. Lu, F. Schlaphof, K. Franke, G. Suchaneck, and G. Gerlach, Integr. Ferroelectr. 62, 13 (2004).

${ }^{8}$ A. C. Busacca, C. L. Sones, V. Apostolopoulos, R. W. Eason, and S. Mailis, Appl. Phys. Lett. 81, 4946 (2002).

${ }^{9}$ C. L. Sones, A. C. Muir, Y. J. Ying, S. Mailis, R. W. Eason, T. Jungk, A. Hoffmann, and E. Soergel, Appl. Phys. Lett. 92, 072905 (2008).

${ }^{10}$ Y. J. Ying, C. L. Sones, H. Steigerwald, F. Johann, E. Soergel, K. Buse, R. W. Eason, and S. Mailis, Proceedings of the Conference on Lasers and Electro-Optics, 2009 (unpublished).

${ }^{11}$ Ferroelectric Crystals for Photonic Applications, 1st ed., edited by P. Ferraro, S. Grilli, and P. DeNatale (Springer, New York, 2008).

${ }^{12}$ T. Jungk, A. Hoffmann, and E. Soergel, J. Microsc. 227, 72 (2007).

${ }^{13}$ M. Jazbinsek and M. Zgonik Appl, Appl. Phys. B 74, 407 (2002).

${ }^{14}$ M. Zgonik, P. Bernasconi, M. Duelli, R. Schlesser, P. Günter, M. H. Garrett, D. Rytz, Y. Zhu, and X. Wu, Phys. Rev. B 50, 5941 (1994).
} 